\title{
Ependimoma Sellar: Reporte de Caso y Revisión de la Literatura
}

\author{
Fuenmayor-Duche Tatiana \\ tatianafuenmayorduche@gmail.com \\ Residente de Neurocirugía, Hospital de Especialidades Eugenio Espejo, \\ Quito-Ecuador
}

Ortiz-Ordonez Andrea

andrea.ortizordonez1989@gmail.com

Residente de Neurocirugía, Hospital de Especialidades Eugenio Espejo,

Quito-Ecuador Universidad San Francisco de Quito USFQ, Colegio de Ciencias de la Salud, calle Diego de Robles s/n y Pampite, Quito 170901, Ecuador

Bucheli-Quezada Paul paulbq1@ hotmail.com Residente de Neurocirugía, Hospital de Especialidades Eugenio Espejo,

Quito-Ecuador Universidad San Francisco de Quito USFQ, Colegio de Ciencias de la Salud, calle Diego de Robles s/n y Pampite, Quito 170901, Ecuador

Quintanilla-González Chrystin chrystinq@hotmail.com Residente de Neurocirugía, Hospital de Especialidades Eugenio Espejo, Quito-Ecuador

\section{Ayala-Ortiz Daniel Alejandro daao1994@gmail.com Universidad Central del Ecuador}

Bottani, Laura Jefe de Servicio de Neurocirugía, Hospital Eugenio Espejo, Quito-Ecuador

\section{RESUMEN}

El ependimoma es una neoplasia neuroectodérmica cuya localización principal es infratentorial en niños y espinal en adultos. Solo se han informado unos pocos casos de localización sellar atípica y no existe un protocolo de diagnóstico o manejo estandarizado para estos casos. Se informa un caso poco frecuente de ependimoma sellar en un adulto con deterioro cognitivo y visual. La sospecha clínica de esta entidad, así como la máxima resección quirúrgica segura, pueden permitir un diagnóstico certero y mejorar el abordaje terapéutico. Se necesitan más estudios sobre este tema para evaluar el tratamiento y la supervivencia a largo plazo.

Palabras clave: Ependimoma intracraneal del adulto, Silla turca, Neurocirugía 
Fuenmayor Duche y otros

Ciencia Latina Revista Científica Multidisciplinar, Ciudad de México, México.

ISSN 2707-2207 / ISSN 2707-2215 (en línea), mayo-junio, 2021, Volumen 5, Número 3. https://doi.org/10.37811/cl_rcm.v5i3.482 p. 2679 


\title{
Sellar Ependymoma: Case Report and Literature Review
}

\begin{abstract}
Ependymoma is a neuroectodermal neoplasm whose main location is infratentorial in children and spinal in adults. Only a few cases of atypical sellar location have been reported and there is no standardized diagnosis or management protocol. A rare case of sellar ependymoma is reported in an adult with cognitive and visual impairment. The clinical suspicion of this entity, as well as the maximum safe surgical resection, may allow an accurate diagnosis and could improve the therapeutic approach. More studies on this topic are needed to assess long-term treatment and over-all survival.
\end{abstract}

Keywords: Adult intracranial ependymoma, Sella turcica, Neurosurgery

Artículo recibido: 05 de Abril 2021 Aceptado para publicación: 28 de Mayo 2021 Correspondencia: aortizo@estud.usfq.edu.ec Conflictos de Interés: Ninguna que declarar 


\section{INTRODUCCIÓN}

El ependimoma (no especificado de otra manera (NOS), epitelial, celular, de células claras, tanicítico, anaplásico, ependimoblastoma) y las variantes como el mixopapilar son raras y representan el $1,9 \%$ de todos los tumores primarios del cerebro y del Sistema Nervioso Central (SNC) en adultos mayores de 20 años. En los niños de 0 a 14 años, representan el 5,7\% de todos los tumores diagnosticados. Para los tumores que afectan la médula espinal, las meninges espinales y la cola de caballo, los ependimomas representan el 20,5\% de todos los tumores diagnosticados en adultos mayores de 20 años y el 21,6\% de todos los tumores diagnosticados en niños de 0 a 19 años. [Ostrom, 2020]

Es una neoplasia neuroectodérmica que nace de las células gliales ependimarias que recubren el sistema ventricular, los plexos coroideos y el canal espinal. La localización extraventricular y sellar es rara (Lima de Barros, Pessoa, Pereira, Moraes, Lima, 2019; Forteza, Pérez, García, Alert, 2018 y Scheithauer, Swearingen, Whyte, Auluck, Stemmer, 2009).

Según la revisión de la literatura en idioma español e inglés, presentamos el caso número 12 reportado hasta el momento de un ependimoma de localización atípica sellar en un adulto que muestra alteración del campo visual y cambios cognitivos (Tabla 1).

Tabla 1. Casos de ependimoma sellar reportados en la literatura.

\begin{tabular}{|l|c|c|c|c|}
\hline \multicolumn{1}{|c|}{ Autor, año } & Edad, sexo & Abordaje & Radioterapia & Seguimiento \\
\hline $\begin{array}{l}\text { Sarkisian \& } \\
\text { Schultz, 1956 }\end{array}$ & $31, \mathrm{~F}$ & Craneotomía & $\mathrm{n} / \mathrm{c}$ & $\mathrm{n} / \mathrm{c}$ \\
\hline $\begin{array}{l}\text { Winer, Lidov } \\
\text { \& Scaravilli, } \\
1989\end{array}$ & $81, \mathrm{M}$ & Transesfenoidal & no & $\begin{array}{c}\text { Fallece por } \\
\text { infección de herida } \\
\text { y meningitis }\end{array}$ \\
\hline $\begin{array}{l}\text { Thomson, } \\
\text { Chakrabarty \& } \\
\text { Marks, 2001 }\end{array}$ & $64, \mathrm{M}$ & Transesfenoidal & si & 3 meses \\
\hline $\begin{array}{l}\text { Chiu et al, } \\
2001 \text {. }\end{array}$ & $32, \mathrm{M}$ & Craneotomía & $\mathrm{n} / \mathrm{c}$ & $\mathrm{n} / \mathrm{c}$ \\
\hline $\begin{array}{l}\text { Mukhida, Asa, } \\
\text { Gentili \& } \\
\text { Shannon, 2006 }\end{array}$ & $43, \mathrm{M}$ & Transesfenoidal & no & 24 meses \\
\hline $\begin{array}{l}\text { Scheithauer et } \\
\text { al, 2009 }\end{array}$ & $71, \mathrm{M}$ & Transesfenoidal & si & 28 años \\
\hline $\begin{array}{l}\text { Belcher, et al, } \\
2010\end{array}$ & $37, \mathrm{~F}$ & Craneotomía & si & 51 meses \\
\hline $\begin{array}{l}\text { Parish, Bonnin, } \\
\text { Goodman \& }\end{array}$ & $46, \mathrm{M}$ & Transesfenoidal & no & \\
\hline
\end{tabular}




\begin{tabular}{|l|c|c|c|c|}
\hline $\begin{array}{l}\text { Cohen-Gadol, } \\
2014\end{array}$ & & si & $\mathrm{n} / \mathrm{c}$ \\
\hline $\begin{array}{l}\text { Ramesh, } \\
\text { Karthikeyan, } \\
\text { Rajaraman, \& } \\
\text { Rao, 2013 }\end{array}$ & $32, \mathrm{M}$ & Transesfenoidal & si & 10 años \\
\hline $\begin{array}{l}\text { Lee, Cho, } \\
\text { Hong \& Shin, } \\
2017\end{array}$ & $59, \mathrm{M}$ & Transesfenoidal & si & 36 meses \\
\hline $\begin{array}{l}\text { Wang S et al, } \\
2017\end{array}$ & $40, \mathrm{M}$ & Transesfenoidal & si & $\begin{array}{c}9 \text { meses, fallece } \\
\text { por infección } \\
\text { respiratoria }\end{array}$ \\
\hline $\begin{array}{l}\text { Fuenmayor, } \\
\text { Ortiz, Bucheli, } \\
\text { Quintanilla y } \\
\text { Ayala, caso } \\
\text { reportado }\end{array}$ & $55, \mathrm{~F}$ & Transesfenoidal & & \\
\hline
\end{tabular}

M: masculino; F: femenino; n/c: no conocido

\section{Reporte de caso}

Paciente de 55 años con antecedente de campo visual alterado de 6 meses de evolución. La resonancia magnética $(\mathrm{RM})$ con contraste mostró una masa multilobular intracraneal heterogénea isointensa $(44 \mathrm{~mm})$ con pequeñas calcificaciones y realce periférico de contraste. La lesión se extendía desde la región sellar hasta la suprasellar desplazando el mesencéfalo, la protuberancia y el quiasma óptico. Además, se informó una ligera dilatación ventricular con leve permeación frontal (Fig. 1).
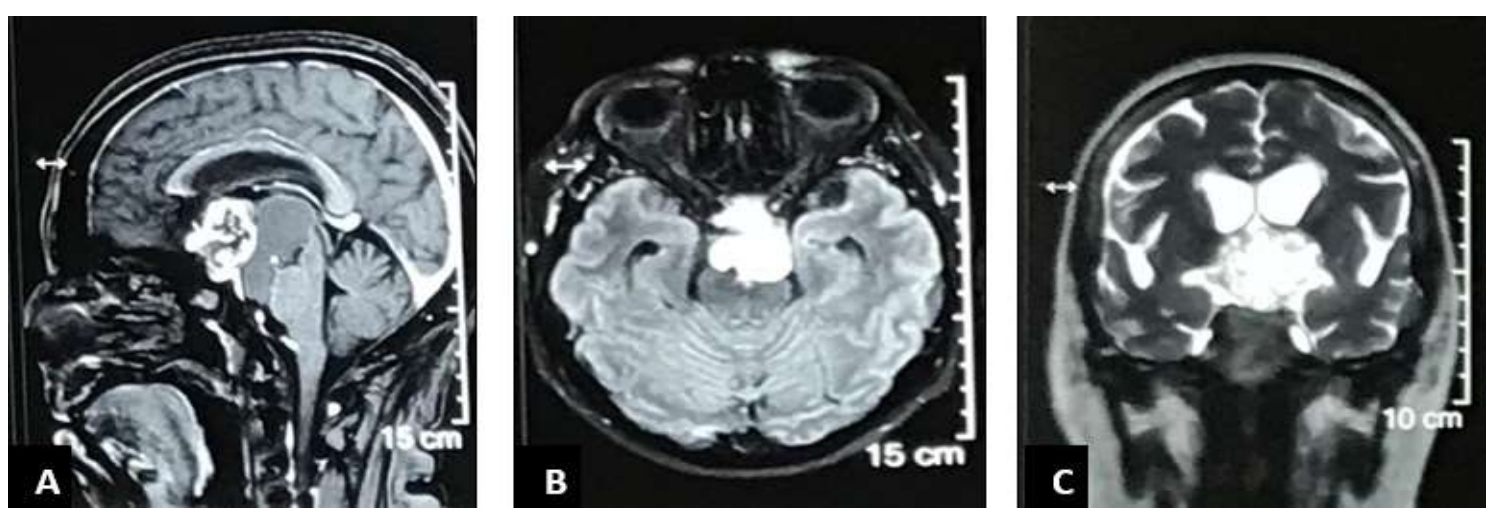

Figura 1. Resonancia Magnética Cerebral. A: Vista sagital, secuencia T1 contrastada; B Vista axial, secuencia FLAIR. C: Vista coronal, secuencia T2.

$\mathrm{Al}$ examen físico, encontramos un paciente letárgico con deterioro de la memoria. El fondo de ojo informó grandes excavaciones y nervios ópticos pálidos; la campimetría confirmó hemianopsia bitemporal. 
Mediante un abordaje neuroendoscópico transeptosfenoidal, se resecó la lesión. Macroscópicamente, el tumor era sólido quístico, grisáceo y con leve vascularización. La gran adherencia a planos profundos dificultó la resección.

El estudio histopatológico reveló estructuras pseudopapilares con empalizada periférica y formación de roseta ependimaria, rodeadas de áreas de hemorragia y tejido fibroso característico del ependimoma grado II de la OMS. El diagnóstico se confirmó por inmunohistoquímica: positivo para GFAP (Proteína Glial Fibrilar Ácida), proteína S-100, Vimentina y EMA (Antígeno Epitelial de Membrana); negativo para Sinaptofisina. (Fig.2)

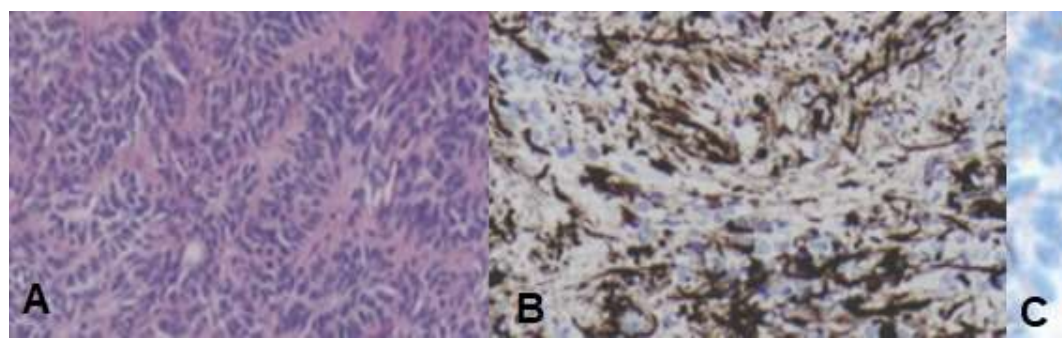

Figura 2. Histopatología e inmunohistoquímica. A: Hematoxilina y Eosina (H\&E) 200x. B: GFAP positiva. C: EMA positiva.

La tomografía computarizada (TC) postoperatoria mostró resección subtotal y pneumocéfalo leve y sin sangrado activo en el sitio quirúrgico (Fig. 3).
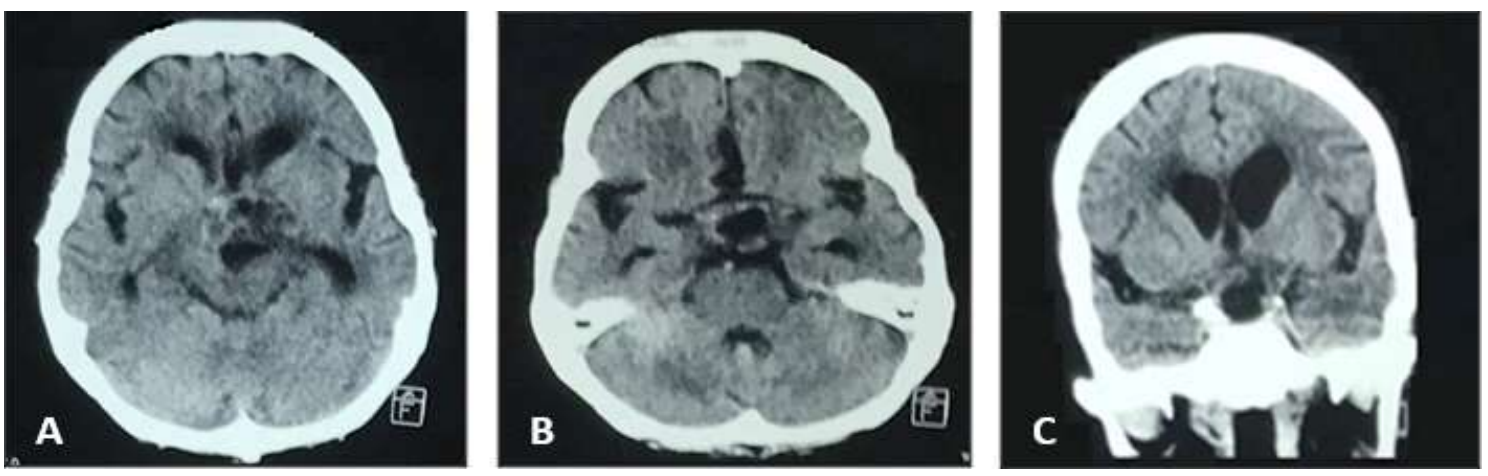

Figura 3. Tomografía Computarizada Cerebral simple. A y B: Secuencia T1, vista axial; $C$ : secuencia $T 1$, vista coronal.

A los 30 días de seguimiento, el paciente informó una mejoría en el campo visual y no se reportó pérdida de LCR (líquido cefalorraquídeo).

La paciente desarrolló hipotiroidismo central y diabetes insípida que fue manejada con desmopresina y hormonoterapia suplementaria. La paciente fue derivada a radioterapia en consideración a una resección tumoral subtotal y grado histológico II. A los 9 meses 
de seguimiento, el paciente falleció por un problema respiratorio dificultando la evaluación de sobrevida global.

\section{DISCUSIÓN}

La etiología del ependimoma sellar no está completamente clara. Podría surgir de restos embriológicos del revestimiento ependimario en el proceso infundibular (Parish et al, 2015). También se ha sugerido una migración aberrante de células neuroectodérmicas primitivas durante el desarrollo embrionario (Scheithauer et al., 2009 y Rodríguez et al., 2017). Dada su ubicación, pueden ser más grandes que los infratentoriales (Mukhida et al., 2006).

Los síntomas dependen de la ubicación, el tamaño y la extensión del tumor; en la región sellar-suprasellar, pueden manifestarse frecuentemente con síntomas visuales, deterioro cognitivo, alteraciones endocrinológicas y cefalea [Parish et al., 2015 y Valassi, Biller, Klibanski \& Swearingen, 2010].

\section{La imagen en el diagnóstico diferencial}

Bravo (2005) afirma que los ependimomas son lesiones muy variables en apariencia radiológica, lo que refleja su variedad histológica y no existen características patognomónicas. Sin embargo, radiológicamente, el comportamiento de los tumores supratentoriales puede diferir de los localizados en otras posiciones. En general, presentan componente quístico (50\%) y calcificaciones (40-80\%) [Rodríguez et al., 2017]. Se requiere de la RM para determinar la ubicación, vascularización, extensión y desplazamiento o compromiso de estructuras neurovasculares para la planificación quirúrgica (Wang et al., 2017 y Bravo et al, 2005).

Generalmente, la RM muestra masas iso-hipointensas respetando la sustancia gris en secuencia T1 y apariencia iso o hiperintensa en secuencias T2. Pueden presentar necrosis o hemorragia intralesional. El realce del contraste suele ser heterogéneo; cuando son homogéneos, pueden simular lesiones extraaxiales como los meningiomas [Bravo et al. 2005 y Cachia et al.,2018].

Rodríguez et al. (2017) indica que el componente quístico a veces no suprime la señal de líquido en FLAIR debido al contenido de proteínas. En las secuencias T2 o de susceptibilidad magnética se pueden observar áreas de baja señal por sangrado o calcificación. La celularidad puede alterar la restricción de la difusión. Sin embargo, según Kuai et al (2020), la restricción de la difusión se consideró una característica de los 
tumores de alto grado y un factor de peor pronóstico para el resultado clínico. Además, la RM con espectroscopía podría mostrar una relación aumentada de Cho/Cr (colina/creatina) y Cho/NAA (colina/n-acetil aspartato) en los ependimomas anaplásicos. La TC sin contraste es útil para identificar componentes quísticos o hemorragia intratumoral (Wang et al., 2017, Rodríguez et al., 2017 y Bravo et al., 2005).

Kuai et al. (2020) informa que en estudios previos han demostrado que la presencia de quistes o necrosis son características de los ependimomas anaplásicos, especialmente los supratentoriales. Además, los quistes mayores de $1 \mathrm{~cm}$ de diámetro se observan también con frecuencia en los supratentoriales, mientras que los infratentoriales tienden a asociarse con neoplasias sólidas.

La lesión podría ser iso o hiperdensa indistinguible de un adenoma hipofisario como en nuestro caso (Wang et al., 2017, Rodríguez et al., 2017 y Bravo et al., 2005).

Según Wang et al. (2017) y Lieberman et al. (2003), la RM con contraste podría ser útil ya que el realce del adenoma es más lento que el de la glándula normal. Por otro lado, el ependimoma se intensifica simultáneamente con la glándula normal. Por tanto, si se observa una lesión selar de naturaleza agresiva y defectos de llenado vascular, puede sugerir ependimoma.

Además, algunos autores recomiendan hacer diagnóstico diferencial con lesiones sólidoquísticas en esta localización como astrocitoma de bajo grado, glioblastoma, tumores neuroectodérmicos primitivos, ganglioglioma, gangliocitoma y oligodendroglioma (Rodríguez et al.,2017, Lieberman et al. 2003 y Wu, Armstrong, Gilbert, 2016).

\section{Histopatología e inmunohistoquímica}

De acuerdo con Villano, Parker, y Dolecek (2013), dado que el entorno clínico y las imágenes por lo general no son concluyentes, el diagnóstico de confirmación es anatomopatológico e inmunohistoquímico. El ependimoma anaplásico es aproximadamente seis veces más frecuente en niños y adolescentes que en adultos, mientras que el patrón inverso es evidente para subependimomas y ependimomas mixopapilares. Para los adultos, el patrón de distribución proporcional para el subependimoma aumenta con la edad, mientras que se observan disminuciones con la edad para el ependimoma mixopapilar.

La histopatología mostrará células ovaladas, alargadas o cuboides en rosetas perivasculares, formadas por agregados de células ependimarias que, si bien no son 
específicas de ependimoma, junto con la positividad de la proteína ácido fibrilar glial permite su diagnóstico, diferenciándola de meningiomas y otros. tumores gliales [Wang et al., 2017 y Bravo et al. 2005).

Wang et al. (2017) afirma que otros marcadores como la positividad para la proteína S100, vimentina y EMA y la negatividad para las hormonas secretadas por la glándula pituitaria y para los neuromarcadores, como la sinaptofisina, confirman el diagnóstico final.

\section{Terapia adjuvante después de la resección quirúrgica}

Zamora y Alkherayf (2020) aseveran que el manejo de los ependimomas sellares no está estandarizado debido al bajo número de casos reportados. Para los ependimomas intracraneales, la cirugía suele ser el tratamiento principal. La resección completa sin enfermedad residual ha presentado mejores resultados clínicos y supervivencia global que la resección parcial.

Se sugiere la resección quirúrgica total o subtotal segura por compromiso de áreas elocuentes o estructuras vasculares, seguida de radioterapia local (Rodriguez et al., 2017, Bravo et al., 2005, Wu et al., 2016 y Hubner, Kool, Pfister, Pajtler, 2018). Igualmente, se recomienda la radioterapia focal o craneoespinal en los casos en que se detecten signos de diseminación tumoral para evitar recidivas (Wang et al., 2017, Wu et al., 2016 y Hubner et al., 2018).

En el caso reportado, al tratarse de una resección subtotal, se derivó a la paciente para recibir radioterapia local con aparentemente buena respuesta a los 6 meses de seguimiento. Sin embargo, al noveno mes el paciente falleció por una infección respiratoria ajena al procedimiento quirúrgico.

Zamora et al. (2020) indica que los ependimomas supratentoriales, a menudo presentan un mayor grado histológico y tienen una menor tasa de supervivencia a pesar del tratamiento con resección y radiación adyuvante.

El papel de la quimioterapia no está bien definido; se menciona su uso en pacientes jóvenes para retrasar la irradiación del sistema nervioso. Sin embargo, no se han realizado estudios prospectivos sobre su eficacia en adultos. Algunos autores recomiendan quimioterapia solo en casos de recidiva [Bravo et al., 2005 y De Tena, 2006). No hay evidencia suficiente para apoyar el uso de quimioterapia [Zamora et al., 2020]

\section{Metástasis a distancia y pronóstico}

Ciencia Latina Revista Científica Multidisciplinar, Ciudad de México, México. ISSN 2707-2207 / ISSN 2707-2215 (en línea), mayo-junio, 2021, Volumen 5, Número 3. https://doi.org/10.37811/cl_rcm.v5i3.482 p. 2686 
Zamora et al. (2020) afirma que las neoplasias malignas primarias rara vez pueden hacer metástasis extracraneal. Los ependimomas intracraneales muestran un bajo potencial metastásico y presentan predominantemente un patrón histológico localmente invasivo. Wu et al. (2016) indica que cuando las metástasis ocurren, generalmente lo hacen en el contexto de la manipulación quirúrgica del SNC y que podrían diseminarse a través de la sangre, la linfa o una derivación ventriculoperitoneal.

Aunque la mayoría de los ependimomas mixopapilares se consideran benignos, pueden tener una recurrencia local o diseminarse a través del líquido cefalorraquídeo. El potencial de diseminación se ha estudiado recientemente y es excepcional que metastatice fuera del SNC (De Tena et al., 2006).

Las metástasis extracraneales ocurren en 0.5 a $1 \%$ de los casos. La diseminación a distancia debe ser considerada cuando luego de la resección quirúrgica tumoral, se requieran cirugías adicionales y/o la colocación de una derivación ventriculoperitoneal [St Jeor, Thacker, Benson, Hull, 2020).

Los sitios más comunes de metástasis extracraneales son los pulmones y los ganglios linfáticos y esto apoya la idea de que la manipulación quirúrgica y el daño de las estructuras del SNC aumentan el riesgo de metástasis (Rodríguez et al. 2017 y St Jeor et al., 2020).

Los autores recomiendan descartar la diseminación a distancia ya que el abordaje quirúrgico de esta entidad suele implicar la manipulación del diafragma sellar y su posible apertura.

Kuai et al (2020) indica que algunos estudios recientes demostraron que los ependimomas supratentoriales exhibieron una supervivencia general y una supervivencia libre de progresión significativamente más deficientes que sus homólogos infratentoriales, lo que sugiere una mayor invasión clínica.

\section{CONCLUSIONES}

El origen del ependimoma sellar no se comprende completamente. Debido al bajo reporte de casos en esta localización, no ha sido posible estandarizar su tratamiento ni el seguimiento. En todos los casos posibles se sugiere la resección total o subtotal segura seguida de terapia adyuvante como la radioterapia local. La manipulación quirúrgica puede aumentar el riesgo de metástasis y si se confirma la apertura dural, los autores sugieren un seguimiento por imágenes para descartar diseminación a distancia. 
Se requieren más estudios para definir el protocolo estándar de manejo, así como conocer la sobrevida libre de enfermedad y global para este grupo de pacientes.

\section{REFERENCES}

Ostrom, Q. T., Patil, N., Cioffi, G., Waite, K., Kruchko, C., \& Barnholtz-Sloan, J. S. (2020). CBTRUS Statistical Report: Primary Brain and Other Central Nervous System Tumors Diagnosed in the United States in 2013-2017. Neurooncology, 22(12 Suppl 2), iv1-iv96. https://doi.org/10.1093/neuonc/noaa200

Lima de Barros Araújo, M., Pessoa Vale, B., Pereira de Macêdo, L., Moraes Sousa, T. and Lima Vale, J., (2019). Ependimoma com recidiva extracerebral intraorbital: relato de caso. Revista Brasileira de Neurologia e Psiquiatria. 23(1):84-92.

Forteza Sáez, M., Pérez Trejo, M., García Socarrás, D., \& Alert Silva, J. (2018). Ependimomas intracraneanos en el instituto nacional de oncología y radiobiología. Revista Cubana de Pediatria, 90(1), 70-78.

Scheithauer, B. W., Swearingen, B., Whyte, E. T., Auluck, P. K., \& StemmerRachamimov, A. O. (2009). Ependymoma of the sella turcica: a variant of pituicytoma. Human pathology, 40(3), 435-440. https://doi.org/10.1016/j.humpath.2008.08.013

Sarkisian, S. S., \& Schultz, A. L. (1956). Pituitary ependymoma. United States Armed Forces medical journal, 7(12), 1813-1816.

Winer, J. B., Lidov, H., \& Scaravilli, F. (1989). An ependymoma involving the pituitary fossa. Journal of neurology, neurosurgery, and psychiatry, 52(12), 1443-1444. https://doi.org/10.1136/jnnp.52.12.1443

Thomson, S., Chakrabarty, A., \& Marks, P. (2001). Ependymoma of the neurohypophysis. British journal of neurosurgery, 15(3), 277-278. https://doi.org/10.1080/026886901750353764

Chiu, V., Chan, C., Chan, P., Chiu, H., Cheung, Y., \& Chan, C. (2001). Suprasellar Ependymoma. Hong Kong Journal of Radiology, 4(2), 169-72.

Mukhida, K., Asa, S., Gentili, F., \& Shannon, P. (2006). Ependymoma of the pituitary fossa. Case report and review of the literature. Journal of neurosurgery, 105(4), 616-620. https://doi.org/10.3171/jns.2006.105.4.616 
Belcher, R., Chahal, H. S., Evanson, J., Afshar, F., Marino, S., \& Grossman, A. B. (2010). Recurrent pituitary ependymoma: a complex clinical problem. Pituitary, 13(2), 176-182. https://doi.org/10.1007/s11102-008-0139-x

Parish, J. M., Bonnin, J. M., Goodman, J. M., \& Cohen-Gadol, A. A. (2015). Intrasellar ependymoma: Clinical, imaging, pathological, and surgical findings. Journal of Clinical Neuroscience, 22(4), 638-641. Retrieved from https://doi.org/10.1016/j.jocn.2014.10.026

Ramesh, V. G., Karthikeyan, K. V., Rajaraman, S., \& Rao, R. (2013). Pituitary ependymoma: Report of a rare case with an insight into the histogenesis. Neurology India, 61(5), 545-546. https://doi.org/10.4103/00283886.121948

Lee, J. S., Cho, K. H., Hong, E. K., \& Shin, S. H. (2017). Pituitary Ependymoma, 10Year Follow-Up after Partial Resection and Radiation Therapy. Brain tumor research and treatment, 5(2), 94-98. https://doi.org/10.14791/btrt.2017.5.2.94

Wang, S., Zong, W., Li, Y., Wang, B., Ke, C., \& Guo, D. (2018). Pituitary Ependymoma: A Case Report and Review of the Literature. World Neurosurgery (Vol. 110). Elsevier Inc. Retrieved from https://doi.org/10.1016/j.wneu.2017.10.134

Rodríguez B, D, Sánchez F, J, Vidal S, N, \& Puyalto. (2017). Caso de desafío diagnóstico. Revista chilena de radiología, 23(1), 2529. https://dx.doi.org/10.4067/S0717-93082017000100006

Valassi, E., Biller, B. M., Klibanski, A., \& Swearingen, B. (2010). Clinical features of nonpituitary sellar lesions in a large surgical series. Clinical endocrinology, 73(6), 798-807. https://doi.org/10.1111/j.13652265.2010.03881.x

Bravo-Bravo, C., Martínez-León, M. I., \& López-Ruiz, P. (2005). Imaging case no. 1. Extraaxial and supratentorial ependymomas. Radiologia, 47(6), 322,354. Retrieved from https://doi.org/10.1016/s0033-8338(05)72864-2

Cachia, D., Johnson, D. R., Kaufmann, T. J., Lowe, S., Andersen, S., Olar, A., Cooper, S. L., Frankel, B. M., \& Gilbert, M. R. (2018). Case-based review: ependymomas in adults. Neuro-oncology practice, 5(3), 142-153. https://doi.org/10.1093/nop/npy026 
Kuai, X. P., Wang, S. Y., Lu, Y. P., Xiong, J., Geng, D. Y., \& Yin, B. (2020). MRI Features of Intracranial Anaplastic Ependymomas: A Comparison of Supratentorial and Infratentorial Lesions. Frontiers in Oncology, 10(July), 1-10. Retrieved from https://doi.org/10.3389/fonc.2020.01063

Lieberman, K. A., Wasenko, J. J., Schelper, R., Swarnkar, A., Chang, J. K., \& Rodziewicz, G. S. (2003). Tanycytomas: a newly characterized hypothalamicsuprasellar and ventricular tumor. AJNR. American journal of neuroradiology, 24(10), 1999-2004.

Wu, J., Armstrong, T. S., \& Gilbert, M. R. (2016). Biology and management of ependymomas. Neuro-oncology, 18(7), 902-913.

https://doi.org/10.1093/neuonc/now016

Villano, J. L., Parker, C. K., \& Dolecek, T. A. (2013). Descriptive epidemiology of ependymal tumours in the United States. British Journal of Cancer, 108(11), 2367-2371. Retrieved from https://doi.org/10.1038/bjc.2013.221

Zamora, E. A., \& Alkherayf, F. (2020). Ependymoma. In StatPearls. StatPearls Publishing. Available from: https://www.ncbi.nlm.nih.gov/books/NBK538244/

Hubner, J. M., Kool, M., Pfister, S. M., \& Pajtler, K. W. (2018). Epidemiology, molecular classification and WHO grading of ependymoma. Journal of Neurosurgical Sciences, 62(1), 46-50. Retrieved from https://doi.org/10.23736/S03905616.17.04152-2

De Tena-Dávila Mata, M. C. (2006). Lumbociatalgia como manifestación de un ependimoma del filum terminal. Rehabilitacion, 40(2), 96-100. Retrieved from https://doi.org/10.1016/S0048-7120(06)74868-X

St Jeor, J. D., Thacker, P. G., Benson, J. C., \& Hull, N. C. (2020). Anaplastic ependymoma metastases though a ventriculoperitoneal shunt. Radiology Case Reports, 15(6), 650-654. Retrieved from https://doi.org/10.1016/j.radcr.2020.02.036 